\title{
Rituximab-associated
}

\section{Hypogammaglobulinemia in pediatric patients with autoimmune diseases}

\author{
Amer M. Khojah ${ }^{1,2^{*}}$ (D, Michael L. Miller ${ }^{1}$, Marisa S. Klein-Gitelman ${ }^{1}$, Megan L. Curran', Victoria Hans ${ }^{3}$,
} Lauren M. Pachman ${ }^{1,3}$ and Ramsay L. Fuleihan ${ }^{2}$

\begin{abstract}
Background: Despite the increased use of rituximab in treating pediatric patients with autoimmune diseases in the last decade, there are limited data on rituximab safety in those subjects who have a developing immune system. The objective of this study is to determine the prevalence of hypogammaglobulinemia in children with autoimmune disease receiving rituximab within the first three years of treatment in the pediatric rheumatology clinic at a tertiary care center.

Methods: We conducted a retrospective chart review of 63 pediatric subjects who received rituximab for the treatment of their autoimmune disease. Immunoglobulin gamma ( $\mathrm{lgG}$ levels, immunosuppressive medication and the need for immunoglobulin replacement therapy were evaluated. Hypogammaglobulinemia was defined as a serum lgG level less than two standard deviations below the mean for age-matched healthy controls.

Results: Twenty-eight patients (44\%) were found to have hypogammaglobulinemia. Hypogammaglobulinemia occurred within the first six months of rituximab treatment in the majority of patients (22 out of 28). The occurrence of hypogammaglobulinemia varied based on the rituximab indication: $46 \%$ pediatric Systemic Lupus Erythematosus (SLE), 71\% autoimmune CNS disease, 60\% ANCA vasculitis, and 12\% in the miscellaneous group. Autoimmune CNS disease had more severe hypogammaglobulinemia, more persistent and was associated with more frequent or severe infections. Three patients with autoimmune CNS disease and one with SLE were given IgG replacement therapy to prevent recurrent or severe infections.

Conclusions: The prevalence of hypogammaglobulinemia in rituximab treated children with autoimmune disease seems to be higher than published data for adults, especially for children with autoimmune CNS disease. The onset of hypogammaglobulinemia is usually within six months of initiation of rituximab therapy. We recommend: 1) obtaining an IgG level prior to starting rituximab; 2) close monitoring for hypogammaglobulinemia after the use of rituximab in pediatric patients and 3) early institution of immunoglobulin replacement therapy if patients develop recurrent infections.
\end{abstract}

Keywords: Rituximab, Hypogammaglobulinemia, SLE, Autoimmune CNS diseases, And ANCA vasculitis

\footnotetext{
* Correspondence: akhojah@luriechildrens.org

${ }^{1}$ Division of Pediatric Rheumatology / Allergy and Immunology, Department

of Pediatrics, Ann \& Robert H. Lurie Children's Hospital of Chicago, 225 East

Chicago Avenue, Box 60, Chicago, IL 60611, USA

2Division of Allergy \& Immunology, Ann \& Robert H. Lurie Children's Hospital

of Chicago, Chicago, IL, USA

Full list of author information is available at the end of the article
}

(c) The Author(s). 2019 Open Access This article is distributed under the terms of the Creative Commons Attribution 4.0 International License (http://creativecommons.org/licenses/by/4.0/), which permits unrestricted use, distribution, and reproduction in any medium, provided you give appropriate credit to the original author(s) and the source, provide a link to the Creative Commons license, and indicate if changes were made. The Creative Commons Public Domain Dedication waiver (http://creativecommons.org/publicdomain/zero/1.0/) applies to the data made available in this article, unless otherwise stated. 


\section{Background}

Rituximab is a chimeric monoclonal antibody directed against CD20, a surface marker on all mature B cells. It leads to depletion of $\mathrm{B}$ cells through several mechanisms which include complement fixation, antibody-dependent cellular cytotoxicity and signaling of apoptosis [1, 2]. Since the drug was approved by the FDA in 1997 for treatment of Non-Hodgkin's lymphoma, its applications have increased tremendously. Rituximab is now used to treat B cell leukemia, various autoimmune diseases, posttransplant rejection, and severe EBV infection [3-6]. The prevalence of hypogammaglobulinemia after rituximab in adult patients with lymphoma is around $40 \%$ with $6 \%$ of these patients requiring IVIG (intravenous gamma globulins) replacement therapy to prevent recurrent infections [7]. However, most of these patients (85\%) received other chemotherapeutic agents which may increase their risk for hypogammaglobulinemia [7]. In a large retrospective study of adult patients with ANCA vasculitis, $26 \%$ of the subjects had hypogammaglobulinemia at baseline and another 30\% developed hypogammaglobulinemia after receiving rituximab. Furthermore, there was a high correlation between the Immunoglobulin gamma (IgG) concentration at the time of rituximab infusion and the nadir IgG concentration post-rituximab [8]. Despite multiple case reports of prolonged hypogammaglobulinemia post-rituximab therapy in pediatric patients, the exact prevalence of this complication in children is less clear [9-11]. A small case series of pediatric patients with Systemic Lupus Erythematosus (SLE) and autoimmune cytopenia revealed that seven out of nine subjects who were treated with rituximab developed hypogammaglobulinemia [12]. This study suggests that pediatric patients are more susceptible to rituximab-associated hypogammaglobulinemia than adults. This may reflect the immaturity of the immune system in children, who have a lower percentage of memory B cells [13]. The aim of this retrospective chart study is to determine the frequency and timing of rituximab-associated hypogammaglobulinemia within the first three years of therapy in children with autoimmune diseases which may lead to an improved screening strategy for this complication.

\section{Methods}

This IRB approved (IRB\# 2015-333) chart review study was conducted at the Ann \& Robert H. Lurie Children's Hospital of Chicago between 2010 and 2019. To standardize monitoring for adverse effects from rituximab, the Pediatric Rheumatology division established an internal guideline in 2015, based on the consensus of all providers (5 attending physicians and a nurse practitioner). These guidelines were applied to patients at baseline and after treatment. Baseline labs included: Complete Blood Count (CBC) with differential, $\mathrm{T}$ and $\mathrm{B}$ cell enumeration by flow cytometry, serum immunoglobulin levels, and vaccine responses to tetanus and Streptococcus pneumoniae antigens before starting of rituximab therapy to rule out primary immunodeficiency such as Common Variable Immunodeficiency. Serum immunoglobulins were measured in the clinical immunology lab using nephelometry. After starting rituximab, follow-up monitoring labs included: $\mathrm{CBC}$ with differential, $\mathrm{T}$ and $\mathrm{B}$ cell enumeration by flow cytometry, and serum IgG levels every three months to monitor B cell reconstitution and exclude hypogammaglobulinemia. In this study, we included all pediatric patients who received a course of rituximab infusions $\left(375 \mathrm{mg} / \mathrm{m}^{2}\right.$ weekly for 4 doses or $750 \mathrm{mg} / \mathrm{m}^{2} 2$ doses separated by 2 weeks) for treatment of autoimmune diseases and had serial serum IgG levels subsequent to rituximab infusions for at least one year. Of note, many subjects received multiple rituximab infusions to maintain long term B cells depletion. We excluded subjects with known primary immunodeficiency diseases or those who needed plasmapheresis or multiple immunoglobulin infusions (IVIG) for treatment of their autoimmune diseases within two years of rituximab initiation. We also excluded patients with baseline hypogammaglobulinemia or patient with significant proteinuria (urine protein to urine creatinine ratio greater than 2). The following variables were extracted from the patient's medical charts for the study period of three years after rituximab therapy: gender, race, and age at time of first rituximab infusion, number of rituximab infusions, other immunosuppressive medications, baseline and follow up immunoglobulin levels, infection history or antibiotic use, need for IVIG, hypoalbuminemia or proteinuria, and history of kidney diseases.

Hypogammaglobulinemia was defined as a serum IgG level less than two standard deviations below the mean for age-matched healthy controls or below $600 \mathrm{mg} / \mathrm{dL}$ in subjects older than 16 years old. Patients with hypogammaglobulinemia were divided into three categories based on the severity. For subjects younger than 16 years old, severity was defined as: mild, IgG level of 2-3 standard deviations below the mean for age-matched controls; moderate, IgG level of 3-4 standard deviations below the mean; severe, IgG level below four standard deviations below the mean. For subjects older than 16 years old, severity was defined as: mild, $400-599 \mathrm{mg} / \mathrm{d}$; moderate, $200-399 \mathrm{mg} / \mathrm{dL}$; and severe, $0-199 \mathrm{mg} / \mathrm{dL}$ [7]. Recurrent or severe infection was defined as three sinus or ear infections per year or hospitalization for severe pneumonia.

IBM SPSS Statistics $25^{\circ}$ software was used to perform Kruskal-Wallis one-way ANOVA for continuous variable and chi-square for the categorical variable to compare the baseline characteristics and hypogammaglobulinemia 
among the various diagnoses. The figures were generated using Graphpad Prism 8 software. The Arthritis Foundation and Children's Arthritis and Rheumatology Research Alliance (CARRA) grant funding was used to measure the serum IgG levels for some of the JDM patients.

\section{Patients}

A total of 63 patients were included in this study. Subjects were grouped according to their diagnoses: 22 with pediatric Systemic Lupus Erythematosus, 14 with autoimmune CNS disease, 10 with ANCA associated vasculitis, and 17 with miscellaneous autoimmune diseases. CNS autoimmune diseases included: 4 with Anti-NMDA receptor encephalitis, 3 with other autoimmune encephalitides, 3 with Opsoclonus-Myoclonus Syndrome, 1 with CNS vasculitis, 1 with Neuromyelitis Optica, 1 with Optic Neuritis, 1 with Chorea. Diagnoses with less than 10 subjects per group were assigned to the miscellaneous group (5 with Juvenile Dermatomyositis, 5 with Polyarticular Juvenile Idiopathic Arthritis, 2 with Mixed Connective Tissue Disease, 2 with Systemic Sclerosis, 2 with Overlap Syndrome, and 1 with Primary Sjogren's Syndrome). Of note, seven of 14 subjects within the autoimmune CNS group received a single trial of IVIG therapy to assess the responsiveness prior to the initiation of rituximab therapy. For the five JDM patients in this study, some serum IgG levels were measured retrospectively using biorepository samples (maintained by Dr. Pachman, collected under IRB \# 2010-14,117) due to lack of IgG measurement at the time of the clinical visit.

Many of the study subjects received additional treatments alongside rituximab therapy. $95 \%$ of the children required a variable amount of steroid treatment over a range of time. Of 63 study subjects, 27 (43\%) were on other immunosuppressive therapy prior to rituximab therapy such as Cyclophosphamide, Azathioprine, Methotrexate, Cyclosporine, Mycophenolate Mofetil, or other biologics, while 23 additional patients received other immunosuppressive medications after beginning rituximab therapy.

\section{Results}

Patient characteristics are presented in Table 1. As expected, the majority of the patients $(86 \%)$ were female. Racial distribution was as follows: $52 \%$ Non-Hispanic Caucasian, 25\% Hispanic, 19\% African American, and $3 \%$ others. The median age of patients with autoimmune CNS disease was 12.4 years which was significantly lower than the other three groups (14-15.9 years) with a $p$-value of 0.026 . Baseline IgG and IgA levels and absolute B cell count were comparable in all four groups, however the IgM level was significantly lower in the autoimmune CNS group. The autoimmune CNS group is expected to have lower immunoglobulins levels because it is consist of younger subjects with lower immunoglobulins reference range . Following rituximab therapy, 28 out of 63 subjects (44\%) had hypogammaglobulinemia. Most of these patients (79\%) developed hypogammaglobulinemia within the first six months of completing rituximab therapy (Fig. 1). The prevalence of hypogammaglobulinemia varied based on the diagnosis: $46 \%$ in pediatric SLE; $71 \%$ in autoimmune CNS disease; $60 \%$ in ANCA Associated Vasculitis; $12 \%$ in the miscellaneous group ( $p=0.006$, Chi-square test) (Fig. 2). Of these 28 with hypogammaglobulinemia, 17 (61\%) persisted for more than six months, with the autoimmune CNS group being disproportionately larger compared to other groups ( $p=0.014$, Chi-square test) (Fig. 2). The severity of the hypogammaglobulinemia varied among the four groups as well, with more severe cases in the autoimmune CNS disease group ( $p=0.035$, Chisquare test) (Fig. 3). Of note, five patients with autoimmune CNS disease, one with SLE, and one with ANCA vasculitis had recurrent or severe infections. IgG replacement therapy was given to all subjects with recurrent or severe infections except three because of parental refusal to start IVIG or management with antibiotics.

\section{Discussion}

Despite the increased use of rituximab in treating pediatric patients with autoimmune diseases in the last decade, there are limited data on the prevalence of rituximab-associated hypogammaglobulinemia, a major adverse reaction reported in the adult literature. In this study, we showed that the prevalence of rituximab-associated hypogammaglobulinemia appears to be much higher in pediatric patients than published data for adults, especially for those with autoimmune CNS disease [7]. This finding could be a consequence of the patient's younger age at the time of rituximab infusion, as the median age of patients with autoimmune CNS disease was significantly lower than the other groups: Five subjects with autoimmune CNS disease were below six years of age. All of these children developed rituximab-associated hypogammaglobulinemia; two required IgG replacement therapy for recurrent or severe infections, one had recurrent infections, but the mother refused IVIG therapy. This finding is consistent with a large multicenter study of rituximab use in pediatric patients with autoimmune CNS disease [14]. The increased susceptibility of rituximab-associated hypogammaglobulinemia in younger children could be explained by their lower baseline IgG levels. Some studies show that IgG levels increase with age up to seven years, while others 


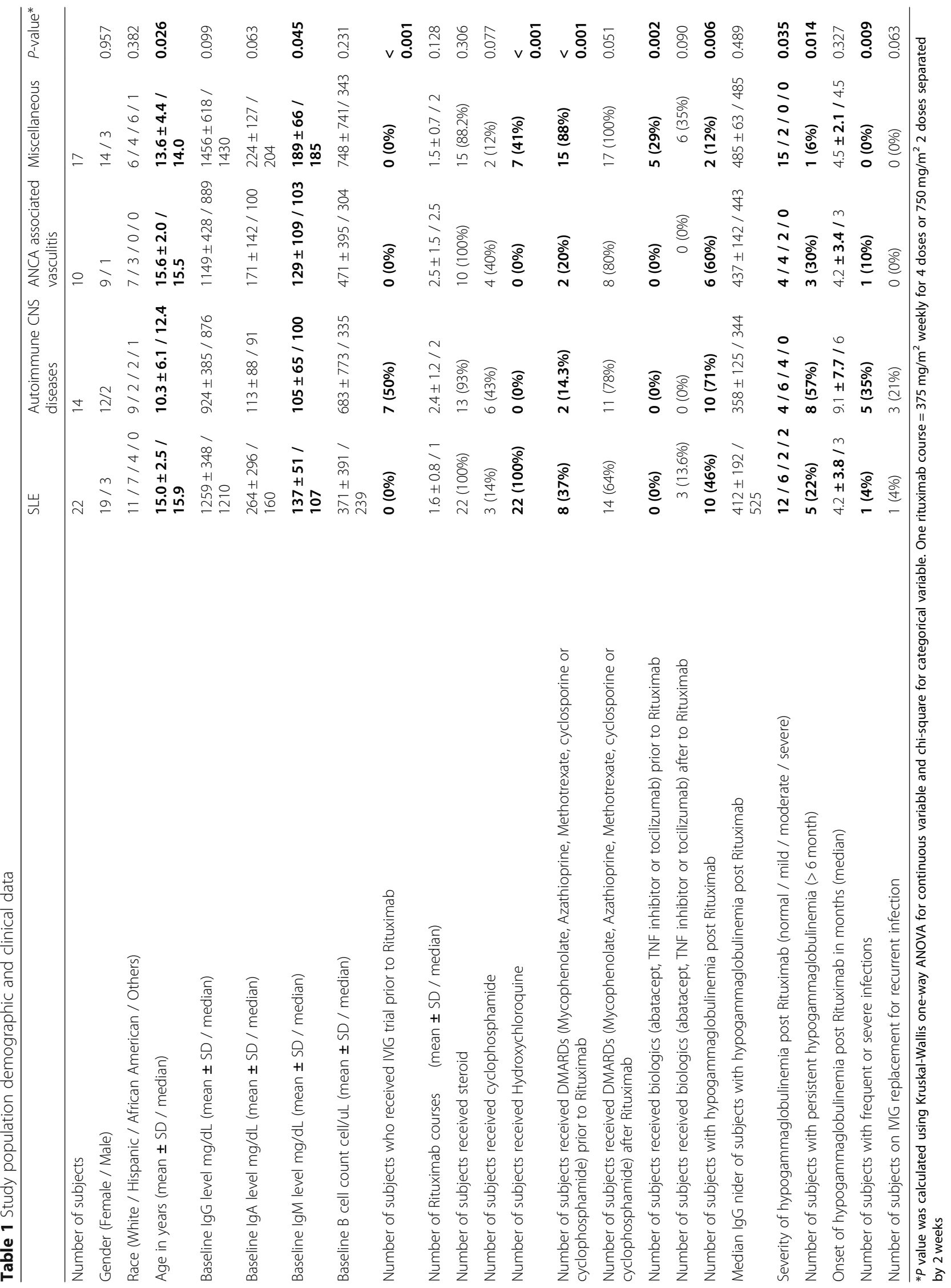




\section{Kaplan-Meier survival curve for the onset of Rituximab-associated Hypogammaglobulinemia}

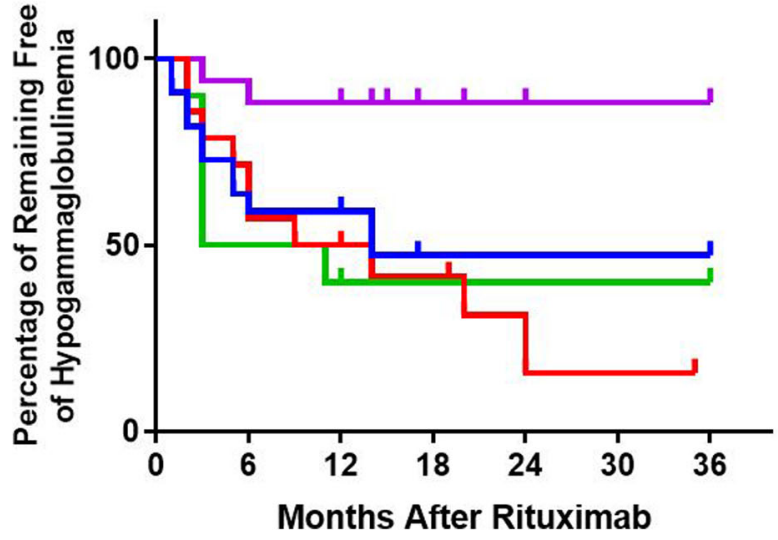

$$
\begin{aligned}
& \perp \text { SLE } \\
& \perp \text { Autoimmune CNS } \\
& \perp \text { Diseases } \\
& \perp \text { ANCA Vasculitis } \\
& \perp \text { Miscellaneous }
\end{aligned}
$$
low Immunoglobulin gamma (lgG) with the first six months of treatment. This difference was statistically significant using the Log-rank test (Mantel-Cox test) $p$-value $=0.02$

show that it takes much longer to reach adult levels $[15,16]$. Long-living plasma cells are the primary source of IgG production, but because of their location within the bone marrow, data about the number of long-living plasma cells in healthy humans is lacking. A murine study showed that the bone marrow stromal cell does not support the development of long-lived plasma cells in very young mice, leading to lower IgG level and an inadequate vaccine response
$[17,18]$. An additional difference in the immune system of adult and children is the higher number of memory B cells in adults compared to children, which are the origin of long-living plasma cells $[13,19]$. Another potential factor that may explain the frequency and severity of hypogammaglobulinemia in patients with autoimmune CNS disease and ANCA vasculitis is the increasing use of cyclophosphamide as adjunctive therapy in 43 and $40 \%$ of the children respectively, which is a known risk factor for

\section{Frequency of Hypogammaglobulinemia}

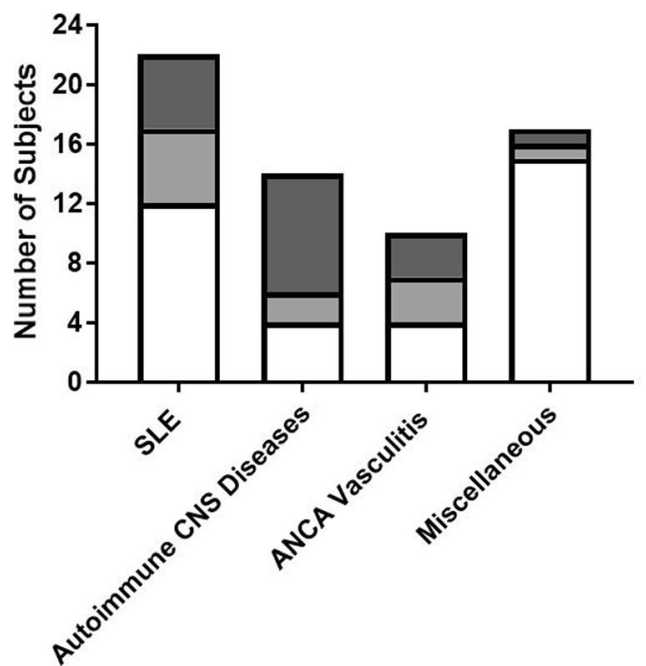

№ hypogammaglobulinemia

Transient hypogammaglobulinemia (less than 6 months)

Persistent Hypogammaglobulinemia (more than 6 months)

Fig. 2 Prevelance of Rituximab-associated hypogammaglobulinemia among various autoimmune diseases. Subjects with hypogammaglobulinemia were divided into two groups-based duration of hypogammaglobulinemia. Transient hypogammaglobulinemia (in light gray) was defined by low lgG for less than six months duration. In contrast, Persistent hypogammaglobulinemia (dark gray) was defined by low lgG for more than six months duration or the need for lgG replacement therapy. Patients with autoimmune CNS disease and ANCA vasculitis had a higher frequency of hypogammaglobulinemia ( $p=0.006$, Chi-square test). Most cases of hypogammaglobulinemia in the autoimmune CNS disease were persistent 


\section{Severity of Hypogammaglobulinemia}

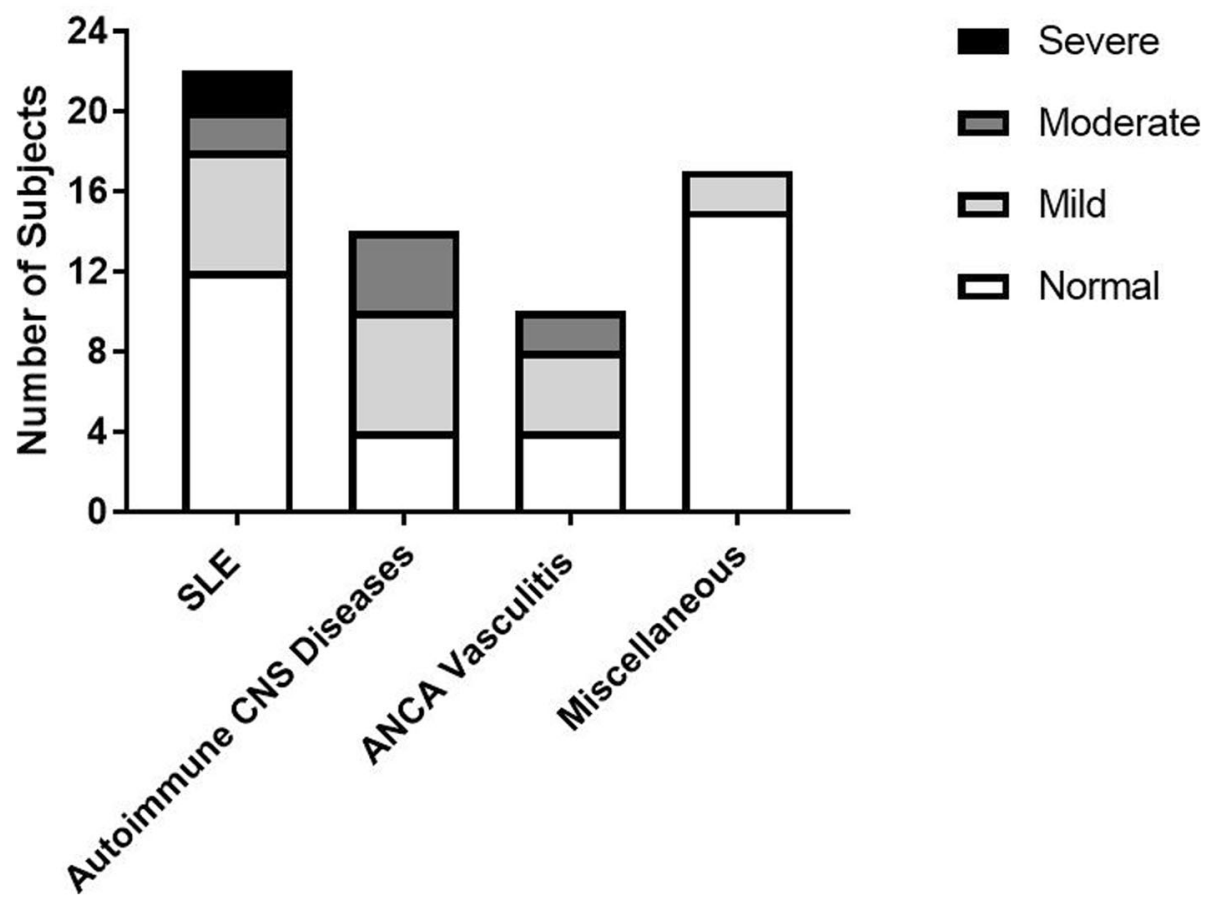

Fig. 3 Prevalence of hypogammaglobulinemia among pediatric patients with autoimmune disease stratified by severity. For subjects younger than 16 years old, severity was defined as: mild, lgG level of 2-3 standard deviations below the mean for age-matched controls; moderate, lgG level of 3-4 standard deviations below the mean; severe, lgG level below four standard deviations below the mean. For subjects older than 16 years old, severity was defined as: mild, 400-599 mg/d; moderate, 200-399 mg/dL; and severe, 0-199 mg/dL

rituximab-associated hypogammaglobulinemia in adults with ANCA associated vasculitis $[8,20]$.

As mentioned earlier, seven of 14 patients with autoimmune CNS disease received a single trial of IVIG therapy prior to starting rituximab. This may explain the observed delayed onset of hypogammaglobulinemia within this group (six months vs. three to 4.5 months of remaining groups) given that the IVIG half-life around three to four weeks [21]. We also noted that autoimmune CNS disease group has a higher percentage of persistent hypogammaglobulinemia ( $80 \%$ vs $50 \%$ of remaining groups) among subjects with rituximab-associated hypogammaglobulinemia.

Previously it has been shown that pediatric SLE patients have a high prevalence of hypogammaglobulinemia [12]. However, we found that many of our SLE patients had significant hypogammaglobulinemia prior to rituximab therapy due to significant glomerular nephritis leading to proteinuria and hypoalbuminemia; therefore, these patients were excluded from this study. Although they were not included, it is important to note that their hypogammaglobulinemia worsened with rituximab therapy and some patients needed IgG replacement therapy. This finding is consistent with data from the use of rituximab in pediatric patients with Idiopathic
Nephrotic Syndrome showed that rituximab prolonged the duration of preexisting low IgG levels [22].

There were a few limitations to this study due to its retrospective nature. Selection bias is possible, which can lead to overestimation of the true prevalence of hypogammaglobulinemia because patients with recurrent or severe infections are more likely to be screened. We tried to minimize this bias by implementing a universal post-rituximab screening strategy in the Rheumatology division which is highlighted in the methods section. Due to the relatively small sample size of the study, the results may not be generalizable and future studies are needed to confirm the findings. Many of the patients in this study received steroids and other immunosuppressive therapy which could affect the immunoglobulin levels. However, this reflects the standard of care treatment of these patients and emphasized the need for routine IgG screening. Another limitation arises from using Lurie Children's Hospital's electronic medical record system to quantify the frequency of infection and antibiotic use. It is possible to miss infections if the patient sought health care elsewhere. However, all patients were advised to notify the rheumatology office if they had a fever or needed antibiotics. Lastly, because autoimmunity can be the only presenting manifestation of a 
monogenic Primary Immunodeficiency disorder, it is difficult to completely rule out this disorder even with normal immunology labs at baseline.

\section{Conclusions}

The prevalence of hypogammaglobulinemia in rituximab treated children with autoimmune disease seems to be higher than published data for adults, especially for children with autoimmune CNS disease. The onset of hypogammaglobulinemia is usually within six months of initiation of rituximab therapy, which can be associated with serious infections. We recommend: 1) obtaining an IgG level prior to starting rituximab, 2) close monitoring for hypogammaglobulinemia after the use of rituximab in pediatric patients, especially in the first six months of patients with autoimmune CNS disease or patients who are receiving cyclophosphamide therapy and 3) early institution of immunoglobulin replacement therapy if patients develop recurrent or severe infections.

\section{Abbreviations}

ANCA: Antineutrophil Cytoplasmic Autoantibodies; CNS: Central Nervous System; IgG: Immunoglobulin G; IVIG: Intravenous Immunoglobulin Therapy; JDM: Juvenile Dermatomyositis; SLE: Systemic Lupus Erythematosus

\section{Acknowledgements}

The authors acknowledge the financial support of Arthritis Foundation and CARRA (Children's Arthritis and Rheumatology Research Alliance) grant.

\section{Authors' contributions \\ AMK: Study design, data collection and analysis, writing the manuscript MLM: Research mentor, help with retrieval of date from EMR, review and edit the manuscript. MSKG: Research mentor, review and edit the manuscript. MLC: Research mentor, review and edit the manuscript. VH: JDM IgG sample collection, review and edit the manuscript. LMP: Research mentor, review and edit the manuscript, PI on the CARRA grant. RLF: Research mentor, review and edit the manuscript. All authors read and approved the final manuscript. \\ Funding \\ Supported by a grant to Dr. Khojah from The Arthritis Foundation and \\ Childhood Arthritis and Rheumatology Research Alliance (CARRA.)}

\section{Availability of data and materials}

Unidentified raw data might be available if requested.

\section{Ethics approval and consent to participate}

This is an IRB approved study (IRB\# 2015-333).

\section{Consent for publication}

The final manuscript has been seen and approved for publication by all the authors.

\section{Competing interests}

The authors declare that they have no competing interests.

\section{Author details}

'Division of Pediatric Rheumatology / Allergy and Immunology, Department of Pediatrics, Ann \& Robert H. Lurie Children's Hospital of Chicago, 225 East Chicago Avenue, Box 60, Chicago, IL 60611, USA. ²Division of Allergy \& Immunology, Ann \& Robert H. Lurie Children's Hospital of Chicago, Chicago, IL, USA. ${ }^{3}$ Cure JM Center of Excellence in Juvenile Myositis (JM) Research and Care, Stanley Manne Children's Research Institute, Chicago, IL, USA.
Received: 7 November 2018 Accepted: 14 August 2019

Published online: 28 August 2019

\section{References}

1. Weiner GJ. Rituximab: mechanism of action. Semin Hematol. 2010;47(2):115-23.

2. Maloney DG. Mechanism of action of rituximab. Anti-Cancer Drugs. 2001;12(Suppl 2):S1-4.

3. Coiffier B, Lepage E, Briere J, Herbrecht R, Tilly H, Bouabdallah R, et al. CHOP chemotherapy plus rituximab compared with $\mathrm{CHOP}$ alone in elderly patients with diffuse large-B-cell lymphoma. N Engl J Med. 2002;346(4):235-42.

4. Edwards JC, Szczepanski L, Szechinski J, Filipowicz-Sosnowska A, Emery P, Close DR, et al. Efficacy of B-cell-targeted therapy with rituximab in patients with rheumatoid arthritis. N Engl J Med. 2004;350(25):2572-81.

5. Becker YT, Becker BN, Pirsch JD, Sollinger HW. Rituximab as treatment for refractory kidney transplant rejection. Am J Transplant. 2004:4(6):996-1001.

6. Patel VL, Mahevas M, Lee SY, Stasi R, Cunningham-Rundles S, Godeau B, et al. Outcomes 5 years after response to rituximab therapy in children and adults with immune thrombocytopenia. Blood. 2012;119(25):5989-95.

7. Casulo C, Maragulia J, Zelenetz AD. Incidence of hypogammaglobulinemia in patients receiving rituximab and the use of intravenous immunoglobulin for recurrent infections. Clin Lymphoma Myeloma Leuk. 2013;13(2):106-11.

8. Roberts DM, Jones RB, Smith RM, Alberici F, Kumaratne DS, Burns S, et al. Rituximab-associated hypogammaglobulinemia: incidence, predictors and outcomes in patients with multi-system autoimmune disease. J Autoimmun 2015;57:60-5.

9. Bisogno G. Persistent B-cell depletion after rituximab for thrombocytopenic purpura. Eur J Pediatr. 2007;166(1):85-6.

10. Castagnola E, Dallorso S, Faraci M, Morreale G, Di Martino D, Cristina E, et al. Long-lasting hypogammaglobulinemia following rituximab administration for Epstein-Barr virus-related post-transplant lymphoproliferative disease preemptive therapy. J Hematother Stem Cell Res. 2003;12(1):9-10.

11. Guerin V, Yakouben K, Lescoeur B, Pedron B, Dalle JH, Baruchel A, et al. Prolonged agammaglobulinemia despite unaltered B-cell lymphopoiesis after peritransplant-rituximab administration in a child. Transplantation. 2008:86(9):1322-3.

12. Kumar S, Benseler SM, Kirby-Allen M, Silverman ED. B-cell depletion for autoimmune thrombocytopenia and autoimmune hemolytic anemia in pediatric systemic lupus erythematosus. Pediatrics. 2009;123(1):e159-63.

13. Morbach H, Eichhorn EM, Liese JG, Girschick HJ. Reference values for B cell subpopulations from infancy to adulthood. Clin Exp Immunol. 2010;162(2):271-9.

14. Dale RC, Brilot F, Duffy LV, Twilt M, Waldman AT, Narula S, et al. Utility and safety of rituximab in pediatric autoimmune and inflammatory CNS disease. Neurology. 2014;83(2):142-50.

15. Buckley RH, Dees SC, O'Fallon WM. Serum immunoglobulins. I. Levels in normal children and in uncomplicated childhood allergy. Pediatrics. 1968;41(3): 600-11.

16. Oxelius VA. IgG subclass levels in infancy and childhood. Acta Paediatr Scand. 1979 Jan;68(1):23-7.

17. Pihlgren M, Friedli M, Tougne C, Rochat AF, Lambert PH, Siegrist CA. Reduced ability of neonatal and early-life bone marrow stromal cells to support plasmablast survival. J Immunol. 2006;176(1):165-72.

18. Haaijman JJ, Van Den Berg P, Brinkhof J. Immunoglobulin class and subclass levels in the serum of CBA mice throughout life. Immunology. 1977;32(6):923-7.

19. Duchamp M, Sterlin D, Diabate A, et al. B-cell subpopulations in children: national reference values. Immun Inflamm Dis. 2014;2(3):131-40.

20. Venhoff N, Effelsberg NM, Salzer U, Warnatz K, Peter HH, Lebrecht D, et al. Impact of rituximab on immunoglobulin concentrations and $B$ cell numbers after cyclophosphamide treatment in patients with ANCA-associated vasculitides. PLoS One. 2012;7(5):e37626.

21. Mankarious S, Lee M, Fischer S, Pyun KH, Ochs HD, Oxelius VA, Wedgwood RJ. The half-lives of igg subclasses and specific antibodies in patients with primary immunodeficiency who are receiving intravenously administered immunoglobulin. J Lab Clin Med. 1988;112(5):634-40.

22. Delbe-Bertin L, Aoun B, Tudorache E, Lapillone H, Ulinski T. Does rituximab induce hypogammaglobulinemia in patients with pediatric idiopathic nephrotic syndrome? Pediatr Nephrol. 2013;28(3):447-51.

\section{Publisher's Note}

Springer Nature remains neutral with regard to jurisdictional claims in published maps and institutional affiliations. 